\title{
LA BANALIDAD DEL MAL Y LA NATALIDAD PENSADAS DESDE EL DESPRECIO Y EL APEGO ${ }^{1}$
}

\author{
Valentina Bulo Vargas ${ }^{2}$
}

\section{Resumen/Abstract}

El presente texto busca ampliar la tesis arendtiana sobre la banalidad del mal, para proponer, de la mano de Heidegger, una aproximación histórica desde donde poder dar cuenta del papel que podrían jugar la afectividad y el cuerpo en ese tema, y en el modo en que son construidas nuestras comunidades. El artículo pretende completar la teoría de Arendt con la afectividad recurriendo a lo que denominaremos desapego absoluto o desprecio, junto a un concepto opuesto, el apego, como complemento a la natalidad propuesta por Arendt. Con ello se busca también abrir la pregunta acerca de la función que podría tener la afectividad en el modo de construcción de nuestras comunidades y, por tanto, de su función política.

Palabras clave: banalidad del mal- afectividad- cuerpo- desprecio-apego

This text seeks to expand Arendt's thesis on the banality of evil, to propose, together with Heidegger, a historical approach to the role that affectivity and body could play on this topic, and furthermore, on the way that our communities are constructed. The article intends to complement Arendt's theory with affectivity, resorting to terms such as absolute disregard or disdain, together with attachment - an opposite concept used to complement what Arendt defines as natality. This is also to open the question of the role of affectivity in the mode of construction of our communities and therefore of its political function.

1 Este escrito forma parte del proyecto de investigación Fondecyt Regular $\mathrm{N}^{\circ} 1130252$ cuya investigadora responsable es Cecilia Sánchez y la autora es co-investigadora.

2 Chilena, Instituto de Estudios Avanzados, Universidad de Santiago de Chile. Email: valentinabulo@gmail.com 

fue lo que le predispuso a convertirse en uno de los mayores criminales de su tiempo...y si bien esto merece ser clasificado como banalidad...., también es cierto que tampoco podemos decir que sea algo normal o común" (Arendt, 1999:136).

En su libro La vida del espiritu Arendt comienza con la alusión al caso de Eichman y su tesis allí enunciada sobre la banalidad del mal; La vida del espiritu, nos dice, busca de algún modo dar fundamento a lo afirmado sobre Eichman; su intención explícita es separarse de ciertas tesis que ligarían el mal al ámbito afectivo, o sea que entenderían el mal a partir de la envidia, el odio, u otros similares. Arendt ve en Eichman el caso ejemplar del mal entendido como incapacidad para pensar (30). Este mal consistiría en el fondo en una deshumanización, tanto por parte de las víctimas deshumanizadas como por parte de los victimarios, que como Eichman no necesitaban ser especialmente perversos o con un odio ideológico sino meramente actuar sin pensar en el contexto del holocausto, y en ese sentido actuar deshumanizadamente, por ello se trataría de un crimen propio contra la humanidad, o de lo propiamente humano. Por otra parte, sin pretender establecer una relación causal, sí es al menos observable la coincidencia en muchos aspectos del no pensar del mal banal con el no pensar epocal heideggeriano, salvo, justamente, por la dimensión afectiva, que quizá fue evitada por Arendt para separarse de una vinculación, llamémosla por ahora "fanática", de pensamiento y afectividad con las consecuencias políticas que ello supone.

En el presente texto nos detendremos en el análisis y la caracterización de la banalidad del mal, como incapacidad para pensar, para proponer complementarla con la dimensión afectiva del pensamiento, en el sentido heideggeriano, específicamente a partir de lo denominaremos desapego absoluto o desprecio. Desde allí podremos vislumbrar también una alusión a la "banalidad de Heidegger mismo" (para citar el libro recientemente publicado de Jean Luc Nancy en octubre del 2015) quien no pudo pensar este desprecio o quizá no pudo salirse de él, como lo muestran nítidamente los "cuadernos negros". Finalmente propondremos retomar la problemática 
idea heideggeriana del nuevo inicio, esta vez pensada junto a la natalidad arendtiana con un componente afectivo y corporal que podría entenderse como apego.

\section{Pensar y no pensar}

El punto de partida para situar la reflexión arendtiana del caso Eichman es pensar la banalidad del mal dentro de una comunidad. Como bien lo indica Cecilia Sánchez, Arendt distingue "entre ser uno (el hombre) y ser varios (los hombres)" y cuando ella habla de deshumanización está entendiendo la pérdida de lo propio de la condición humana, de la pluralidad, es decir del estar en el mundo de los hombres unos con otros.

La banalidad del mal se determina por pensar o no pensar, pues el pensar para Arendt ocupa un lugar central no sólo en lo que podríamos llamar "las estructuras de los hombres" sino que cumple una función determinante en el curso de la historia y la construcción de comunidades, o sea, en el ámbito político. Aunque el texto de Eichman se apega al caso y a una descripción de su contexto, lo cierto es que Arendt indica claramente hacia una responsabilidad política de la toda la comunidad europea en la ejecución de la solución final. El holocausto no obedece entonces solamente a un fallo de algunos, o de un cierto sector sino que se trataría de la conformación de lo que podríamos llamar una comunidad del no pensamiento, que constituiría el desmoronamiento moral y colapso de la sociedad europea.

Sin excusar a Eichman, Arendt intenta mostrarnos que es la comunidad europea en cuanto tal la que se deshumaniza: obviamente por parte de los alemanes, Arendt dice que "la práctica del autoengaño se extendió tanto, convirtiéndose casi en un requisito moral para sobrevivir" (Arendt, 1999: 31) y que "del conjunto de pruebas de que disponemos solamente cabe concluir que la conciencia, en cuanto tal, se había perdido en Alemania" (Arendt, 1999: 51). La opinión pública llega incluso a pensar que en caso de derrota en la guerra "el Führer, en su gran bondad, tiene preparada para todo el pueblo alemán una muerte sin dolor, mediante gases, en caso de que la guerra no termine con nuestra victoria” (Arendt, 1999: 63). Pero no sólo se trata de los alemanes considerados individualmente sino que también la institucionalidad, el aparato estatal cumple una función determinante pues "la solución final, si quería aplicarse a la totalidad de Europa, exigía algo más que la tácita aceptación de la burocracia del Reich, exigía la activa 
cooperación de todos los ministerios y de todos los funcionarios públicos de carrera” (Arendt, 1999: 65).

Pero Arendt va más allá de responsabilizar a los que son considerados victimarios. Las controvertidas afirmaciones que hace respecto a la comunidad judía apuntan en la misma dirección; "para los judíos, el papel que desempeñaron los dirigentes judíos en la destrucción de su propio pueblo constituye uno de los más tenebrosos capítulos de la historia de los padecimientos de los judíos en Europa...en los manifiestos que daban a la publicidad, inspirados pero no dictados por los nazis, todavía podemos percibir hasta qué punto gozaban estos judíos con el ejercicio del poder recientemente adquirido" (Arendt, 1999: 75). Además, "la determinación de los individuos que debían ser enviados a la muerte, era, salvo excepciones, tarea de la administración judía” (Arendt, 199: 79).

Arendt afirma explícitamente el "colapso moral que los nazis produjeron en la respetable sociedad europea, no sólo en Alemania, sino en casi todos los países, no solo entre los victimarios, sino también ente las víctimas" (Arendt, 1999: 87). Este colapso tiene que ver con el no pensar y por ello con la deshumanización tanto como "ataque a la diversidad humana como tal, es decir, a una de las características de la 'condición humana" (Arendt, 1999: 110) como por aquello que "es esencial en todo gobierno totalitario, y quizá propio de la naturaleza de toda burocracia, transformar a los hombres en funcionarios y simples ruedecillas de la maquinaria administrativa, y en consecuencia, dehumanizarles" (Arendt, 1999: 139).

En estas citas aparece el sentido concreto que tiene para Arendt no pensar: "no tuvo Eichman ninguna necesidad de 'cerrar sus oídos a la voz de la conciencia'...debido no a que no tuviera conciencia, sino a que la conciencia hablaba con voz respetable, con la voz de la respetable sociedad que le rodeaba" (Arendt, 1999: 88). El mal de Eichman, para Arendt consiste en "su incapacidad para hablar que iba estrechamente unida a su incapacidad para pensar, particularmente para pensar desde el punto de vista de otra persona. No era posible establecer comunicación con él, no porque mintiera, sino porque estaba rodeado por la más segura de las protecciones contra las palabras y la presencia de otros, y por ende contra la realidad como tal" (Arendt, 1999: 27).

Podemos decir desde ya que para Arendt el mal banal consiste en no pensar, y específicamente en esta cita, en no ponerse en el punto de vista del otro. Cabe hacer notar que la dimensión afectiva del pensar no 
aparece tematizada aquí, justo cuando se destaca la función del pensar en términos políticos, vale decir, en lo que respecta a las relaciones "entre" unos y otros. Adelantando un poco nuestra tesis diremos que el mero no ponerse en el punto de vista del otro ha de ser complementado con una dimensión afectiva para caracterizar al mal banal, pues ponerse en el punto de vista de otro se puede hacer de muchas maneras, al menos es posible, por ejemplo para un perverso, hacer el mal poniéndose en el punto de vista del otro, justamente para hacerle dańo; proponemos que es necesario agregar una tonalidad específica que indique el sentido de la vinculación con el punto de vista del otro.

En La vida del espiritu, Arendt precisa que el pensar es junto a la voluntad y el juicio una de las tres actividades mentales básicas y "aunque no puede cambiar jamás la realidad de un modo directo, de hecho en nuestro mundo no hay oposición más clara y radical que entre hacer y pensar, los principios a partir de los que se actúa y los criterios a partir de los cuales se juzga y se conduce la vida dependen, en última instancia de la vida del espíritu. En realidad, la ausencia de pensamiento es un factor poderoso en los asuntos humanos" (Arendt, 2002: 93). Esto quiere decir que el pensamiento tiene una función política determinante y también que es la condición que lleva a los humanos a evitar el mal y buscar el bien (Arendt, 2002: 31). Es una función política porque está vinculada al modo de relaciones que se pueden dar entre los hombres y justamente "la política trata del estar juntos y los unos con los otros de los diversos. Los hombres se organizan políticamente según determinadas comunidades esenciales en un caos absoluto, o a partir de un caos absoluto de las diferencias" (Arendt, 2013: 45).

Tanto en La vida del espiritu como en La condición humana el pensamiento es una actividad del espíritu que aunque no es directamente una acción sí la determina de modo fundamental, en cambio cuerpo y afectividad están más del lado de lo pasivo. "El alma, donde emergen nuestras pasiones, sentimientos y emociones, es una mezcla más o menos caótica de hechos que no creamos sino que sufrimos....su invisibilidad recuerda a la de los órganos corporales internos...la vida del espíritu, por el contrario es pura actividad, que puede iniciarse o detenerse a voluntad..., la única manifestación del espíritu es la distracción" (Arendt, 2002: 94ss). Por otra parte, "lo que es objeto de los sentidos se llama 'cuerpo" (Arendt, 2002: 95). Como afirma Cecilia Sánchez, entonces, Arendt "no admite en la comunidad plural la vida íntima, las pasiones del corazón, los pensamientos de la mente o los placeres sensoriales: los tacha de inapropiados porque 
carecen de un lenguaje singularizador (Arendt, 2202: 14).

Podría parecer un contrasentido plantear una función política del pensamiento siendo que éste en tanto inmaterial ser "resta" de la contingencia, incluso Arendt llega a afirmar que "el yo pensante es pura actividad y, por lo tanto, no tiene edad ni sexo, carece de cualidades y de biografía"(Arendt, 2002: 67). Justamente la labor del pensamiento tiene que ver con conducir la acción y el medio de esta conducción es el lenguaje. Las actividades mentales se conciben discursivamente y en relación esencial con el lenguaje, y es en el lenguaje que se da fundamentalmente este "entre" uno y otro que constituye lo político. Justamente no pensar tiene que ver con dejarse llevar por las pasiones o el cuerpo. "En lo que se refería a Eichman, todo dependía de las variaciones del estado de ánimo” (Arendt, 1999: 35), justamente ello es prueba del no pensar.

\section{Función politica de la afectividad a partir de Heidegger}

Como dijmos al principio del texto, en su enunciación, el diagnóstico de Arendt coincide exactamente con el de Heidegger: estamos faltos de pensamiento, o mejor, nuestro mundo se caracteriza epocalmente por estar faltos de pensamiento. En ambos autores esta afirmación se dice respecto a una comunidad en un tiempo determinado, porque tiene que ver con ciertas condiciones de posibilidad de relaciones entre unos y otros las que no dan de sí para que haya pensamiento. Una de las grandes diferencias, además de la conducción a la acción del pensamiento en Arendt, es la inclusión predominante por parte de Heidegger de la afectividad (a través de los llamados temples fundamentales) en el pensamiento y su carácter histórico, y por ende un rol fundamental a la hora de conformar comunidad ${ }^{3}$. Quizá sea este punto precisamente lo que lleva a Arendt a querer distanciarse de una lectura "afectiva" del pensamiento y su función política, porque es posible que la lea como un cierto peligro a las derivas "mesiánicas" en las que Heidegger incurrió, en las que precisamente Arendt no quiere caer. Los Cuadernos Negros nos muestran hoy la vinculación explícita que hace Heidegger de esta figura epocal con el pueblo judío:

"Los judíos "viven" por su aptitud particularmente acentuada para calcular (bei ihrer betont rechnerischen Begabung), ya desde hace mucho tiempo según el principio de la raza (Rasse-prinzip); razón por la cual ellos

3 He trabajado esta temática con detenimiento en Bulo 2012. 
se defienden también violentamente contra su aplicación ilimitada. La instauración de la crianza racial no proviene de "la vida" en cuanto tal, sino de una supremacía de la vida a través de la maquinación. Lo que trama ésta última a través de una planificación semejante es la desracialización completa de los pueblos, mediante la fijación en la instauración uniformemente construida y recortada de todo ente. Con la desracialización va a la par una auto alienación de los pueblos - la pérdida de la historia- es decir, de los ámbitos de decisión para con el Ser" (Heidegger, 2014: 82)

Abordaremos nuevamente esta cita al final del escrito, antes es necesario bosquejar algunos trazos sobre las figuras epocales de la historia del ser y la función que cumplen allí las tonalidades afectivas:

Imaginemos un momento de nuestra historia en que el horizonte de comprensión se estrecha y literalmente no está la posibilidad de distinguir cómo salir o cómo se ha entrado en esa estrechez o angostura de posibilidades de comprensión y decisión, es parecido a lo que vemos en esas películas de ficción donde hay túneles donde el espacio-tiempo se estrecha. Heidegger denomina apremio (die Not) a este momento, que en cierta medida expresa el que determinadas posibilidades históricas estén agotadas y es necesario el nacimiento de nuevas vías, pero éstas aún no se vislumbran porque no está el espacio-tiempo para que surjan siquiera como posibilidad. El apremio es la condición de posibilidad de que pueda resurgir nuestro mundo desde un horizonte distinto; otro inicio. En esta angostura, que es pura tensión, es donde se gatilla un inicio histórico, es por ello también una necesidad y una urgencia histórica, porque fuerza un inicio.

Del apremio pueden surgir distintas tonalidades que determinarán el abanico y el curso de posibilidades históricas; son para Heidegger los llamados temples fundamentales o "rectores" porque tienen la fuerza de poder reconfigurar nuestro modo de relacionarnos los unos con los otros. Un tono determina un cierto campo de oscilación, en el que se delimita nuestro horizonte de comprensión del mundo. La propuesta heideggeriana de los temples fundamentales no debe ser confundida con un irracionalismo o

4 "Die Juden »leben" bei ihrer betont rechnerischen Begabung am längsten schon nach dem Rasseprinzip, weshalb sie sich auch am heftigsten gegen die uneinge- schränkte Anwendung zur Wehr setzen. Die Einrichtung der rassischen Aufzucht entstammt nicht dem »Leben« selbst, sondern der Übermächtigung des Lebens durch die Machenschaft. Was diese mit solcher Planung betreibt, ist eine vollständige Entras-sung der Völker durch die Einspannung derselben in die gleich- gebaute und gleichschnittige Einrichtung alles Seienden. Mit der Entrassung geht I eine Selbstentfremdung der Völker in eins - der Verlust der Geschichte - d.h. der Entscheidungsbezirke zum Seyn”. 
pensada como un orden del corazón escindido del pensamiento; los temples fundamentales son históricos (en rigor, ontohistoricos) porque configuran las relaciones fundamentales de una época y son una misma cosa con el pensamiento, son la forma o el estilo del pensar de una época.

Heidegger sitúa las tonalidades afectivas, al menos algunas, en un plano que trasciende lo subjetivo, los temples aquí no son el aspecto subjetivizante de un pensamiento sino su fundamento ontológico, ya que cada época estaría regida y configurada desde un tono sobre el cual ciertas lógicas adquieren sentido. Son el tono de la voz del Ser. Es imposible, lo anuncia Heidegger tempranamente, que exista pensamiento sin tonalidad afectiva (Heideggeer, 1986: 134), y esto es más radical que decir que el sujeto que piensa está bajo el influjo de algún sentimiento, Heidegger se refiere al pensamiento mismo, a las ideas, las teorías, los conceptos, y hasta las percepciones, ellos tienen tonalidad afectiva y no pueden no tenerla. Para que nosotros podamos percibir algo, un poste de luz, por ejemplo, primero debe estar bosquejada la posibilidad de que me pueda llegar a la presencia; hay algunos tonos desde los cuales sencillamente "no vemos" el poste de luz. Por eso todo pensamiento tiene un tono: una fórmula química, un formulario de atención médica, poseen tono, fuerza y marcan una distancia, no hay pensamiento neutro. Heidegger analiza distintos tonos epocales como el asombro griego, la certeza moderna, el duelo sagrado, el aburrimiento, la angustia, el espanto, entre otros, y en cada uno va mostrando el modo que este tono determina las lógicas imperantes de cada época, las relaciones fundamentales y con ello el modo de acontecer la verdad, o sea lo que nos aparece como "naturalmente" verdadero, justamente porque está dicho en determinado "tono".

La pregunta que se hace Heidegger $-y$ que retomaremos luego junto a Arendt para realizar una propuesta conjunta- es por el tono que posibilitaría un reinicio de nuestra historia, de modo de poder fundamentar otras posibilidades, y con ello literalmente construir otro mundo. Heidegger sabe que no se puede forzar esa situación, porque justamente no se puede manipular al antojo un temple, no se trata de decir, por ejemplo, bueno, ahora confío. Aún así Heidegger trata de imaginar cómo tendría que ser un tono que supere nuestro estado de dominación científico técnica y que sea capaz de abrirnos otro horizonte de comprensión y nos dé otro fundamento de verdad. Este intento lo realiza con distintos nombres en distintos textos, pero es sin duda el desasimiento (o serenidad) el que ocupará el sitial de la angustia del primer Heidegger y podría establecerse como el tono fundamental de un posible segundo inicio histórico. Volveremos a esto al 
final de nuestro escrito.

Aunque no de un modo explícito, podemos identificar claramente en Heidegger una función (onto) política de la afectividad ya que, como hemos visto, los temples fundamentales no son puramente subjetivos, ni pasivos e irracionales sino que son la forma misma del pensamiento en una determinada época desde donde se configura un horizonte de comprensión y en este sentido la conformación de una comunidad. Por otra parte, es el pensamiento mismo el que posee un tono afectivo, si se acepta esta tesis no hay un pensamiento afectivamente neutro y por tanto se hace necesaria la pregunta por la dimensión afectiva de cada pensamiento.

\section{Desasimiento, apego y desprecio: función politica de la afectividad}

Es desde las tesis heideggerianas sobre los temples fundamentales que hemos expuesto que podemos proponer complementar la definición arendtiana de la banalidad del mal con una tonalidad afectiva específica, con ello apuntamos también hacia la necesidad de identificar las distintas funciones que puede tener la dimensión afectiva en el ámbito político. Para mostrar esto un poco más claramente, indicaremos algunas tonalidades que podrían "completar" la tesis arendtiana sobre la banalidad del mal, estas son el desprecio, la ira y el odio que actuarían como fundamento del horizonte de posibilidades en determinados momentos históricos.

En primer lugar, el odio, según Arendt, constituye "esta desintegración de la vida política, este odio vago y penetrante hacia todos y hacia todo, sin un foco para su apasionada atención y nadie a quien responsabilizar de la situación: ni al Gobierno, ni a la burguesía, ni a una potencia exterior" (Arendt 1999b: 225). Aquí se ve que para Arendt hay una función de las pasiones en la vida política, en este caso para desintegrarla. Jacques Rancière, en una reflexión cercana a esta, llega a hablar incluso de un odio reunidor, que consiste en un "reunir para excluir...el ladrido de la jauría" (Rancière, 2007: 46s). Este odio no se explicaría sólo por la disputa entre unos y otros por algún bien o el miedo a perderlo, no se explicaría sólo desde una privación real o posible sino que estaría en su base el simple rechazo del otro (Rancière, 2007: 46-55). El odio puede estar en la base de una comunidad y desde allí puede operar la exclusión, "el tercero excluido, el entre-dos impensado es la socialización del odio, la comunidad que se forma no para apropiarse de los bienes del otro sino simplemente por y para el odio"(Rancière, 2007: 52). Esta tesis se dirige, como lo sugiere Rancière, a fundamentar el "mal 
radical" y dar explicación de la catástrofe totalitaria, y es una especie de punto ciego para la filosofía, ya que "le cuesta abordar: ese punto en que el orden de la jauría de las reuniones populares, esa articulación de lo uno y lo múltiple que no es ni reunión de lo múltiple discordante ni regulación del litigio, sino el punto en que los terrores de cada uno coinciden con aquellos de lo múltiple; en que la angustia del sujeto desposeído deviene fuerza de odio avasallador, en el que el remedio a la separación se convierte en mal radical" (Rancière, 2007: 52ss).

Muy distinto es el caso de la ira, que Ernst Bloch denomina pasión roja a diferencia del odio que sería una pasión amarilla. "El odio es pálido, encogido, cobarde, pestífero, encierra vapores de cerveza que pueden ser muy explosivos. La ira es abierta. No hace empalidecer, sino enrojecer. La ira olvida las precauciones, a diferencia del desgraciado y pequeñoburgués nazi que adopta todas las precauciones. La ira no es oportunista; puede estallar repentinamente, contra el interés propio, y por ello tiene parentesco con cosas más elevadas...todas las revoluciones han ido acompañadas por la ira" (Häsler, 2002: 16). La ira se distingue también del desprecio, pues es más bien es una reacción ante el desprecio que se siente como injustificado. Una de las reacciones ante el desprecio, que justamente consiste en desplazarlo haciendo irrumpir la fuerza de otro es la indignación del despreciado. La ira, como lo afirma Aristóteles en La Retórica es un "deseo triste de dar un castigo manifiesto por un desprecio no merecido" (Aristóteles, 1999: 96). Aristóteles nos dice que a los inferiores no les corresponde despreciar, lo que causa más ira en ellos porque no son tenidos en ninguna estimación.

El desprecio es una figura que curiosamente no vio Heidegger ni siquiera después del holocausto y que emerge no de la falta de apremio - como el aburrimiento- sino de la falta de apego. ¿Podríamos pensar un mundo articulado a partir del desapego absoluto? ¿una especie de desprecio articulante?

El desprecio más bien se caracteriza por un "no sentir" (Aristóteles, 1999: 100); el desprecio no odia ni teme porque no hay otro que aparezca como tal en el horizonte. El desprecio opera como un modo de construcción de identidad a partir del no reconocimiento del otro: soy lo que soy justamente a partir de que el otro no sea otro. Marie France Irigoyen trabaja este punto en su análisis del perverso (Irigoyen, 1999, 99ss); la figura de narciso es muy decidora, porque en la lógica perversa no hay ningún otro. El desapego absoluto es justamente el no reconocimiento del otro en tanto que otro, y desde ese horizonte ni siquiera puede abrirse la posibilidad 
de que aparezca. Irigoyen nos dice que no hay manera de ganarle a un narciso perverso, a menos de ser o convertirse en alguien más perverso que él. Ciertos momentos históricos, desde este análisis, podrían explicarse como una cristalización o acumulación de desprecio, y aunque, como afirma Betancourt-Fornet que "en la exigencia ética del reconocimiento del otro está latente una dialéctica cuyo conflicto de fondo remite a un hecho consumado por la historia de inhumanidad escrita hasta hoy, a saber, que la negación del otro ha funcionado como un "pretexto" para ocupar el mundo del otro, para invadir su espacio y su tiempo, para declarar en fin "desiertas" sus almas y sus mundos de vida" (Betancourt-Fornet, 2011: 23), sin un apego pensado como condición básica para abrir la posibilidad de un comienzo histórico desde el reconocimiento mutuo difícilmente podremos desplazar o remover nuestras lógicas despreciantes.

Si pensamos en Eichman pareciera que es el desprecio el que tonaliza su incapacidad para pensar, como observa Arendt no es la ira u el odio sino esa incapacidad para ponerse en lugar del otro, y agregamos aquí, ese temple que impide cualquier reconocimiento de otro, porque ni siquiera puede aparecer como otro. Parece al menos viable pensar también en un desprecio comunitario o conformador de comunidad, donde justamente se constituye la "identidad de un pueblo" a partir de la negación de otro. Si pensamos en Heidegger, en la filosofía de Heidegger la pregunta recae en la historia del ser y es allí donde habría que buscar ciertos atizbos de odio, ira o desprecio.

\section{El problema del segundo inicio}

En su libro titulado La banalidad de Heidegger Nancy afirma que "Heidegger ha retomado las fórmulas más violentas de esta doxa integrándolas a un sistema de pensamiento: los Judíos finalizarían el derrumbe de occidente . Eso era evidente. Sabíamos que él era, como tantos otros, antisemita. No habíamos leído las notas de esos cuadernos negros que entraban en la infamia de todo un aspecto de su pensamiento. No el pensamiento "del ser" sino aquel de una historia-destino y del deseo feroz de un "nuevo inicio". Inaugurar, fundar, ser en lo inicial, viejos pruritos metafísicos” (Nancy, 2015: 7).

¿Está el problema, como dice Nancy, en la obsesión por un reinicio de la historia, en la pretensión, por lo demás muy antigua, de fundar un nuevo mundo estableciendo violentamente una especie de "página en blanco" donde reescribir todo otra vez desde cero? 
Las afirmaciones de Peter Trawny son bastante más radicales porque él sí vincula el antisemitismo heideggeriano a la historia del ser: "la filosofía ha abierto su pensamiento a un antisemitismo que puede ser llamado más precisamente un antisemitismo inscrito en la historia del ser" (Trawny, 2012: 26), donde, como bien señala Marcos García de la Huerta, los griegos son al primer inicio como los alemanes al segundo (2014: 221), y la judería (Judentum), como dice despectivamente Heidegger en reiteradas ocaciones constituye la figura de la decadencia y final del abandono del Ser, la Maquinación, de ahí que "la aptitud tenaz para el cálculo, el tráfico y la confusión sobre las cuales estaría fundada la ausencia de mundo de la judería" (García de la Huerta, 2015: 223).

Lo que no agrega Trawny es que la figura del nacionalsocialismo está igualmente incrustada para Heidegger en la Maquinación: "posiciones como 'nacionalismo' y 'socialismo' pertenecen a un período en el que la edad moderna permanece todavía en la etapa preliminar de su finalización; ahora sólo se utiliza el término histórico para un sentido totalmente diferente, a esto ya no se le puede llamar 'política" (Heidegger, 2014: 43).

François Fedier afirmará, en el extremo contrario, en defensa de Heidegger, que las afirmaciones de que Heidegger es antisemita no tienen fundamento : "leyendo el pequeño libro de Peter Trawny, constatamos que, sobre la base de una interpretación caricaturesca, más exactamente, empecinándose desde un comienzo sobre la incomprensión, eso que dice Heidegger está con todas sus letras trampeado, para luego, revisando algunos textos cuidadosamente sacados de contexto, imputar al filósofo un antisemitismo fundamental" (Fedier, 2014:11).

Hay un punto ciego respecto de esta cuestión que no dan cuenta ni Fédier, ni Trawny respecto al problema del segundo inicio y su vinculación estructural al antisemitismo, y como vimos tampoco está presente en la tesis de Arendt sobre la banalidad del mal y es lo que ha querido ser tematizado en este escrito: la tonalidad de la historia. Desde aquí, me parece puede reelaborarse la pregunta respecto al segundo inicio heideggeriano así como respecto a la banalidad del mal y del mal de Heidegger.

Nos acercaremos ahora a las tonalidades contrarias a las que hemos visto, que en términos históricos responden justamente a la pregunta por la posibilidad de iniciar otra vez nuestra historia, de abrir el campo de posibilidades, nos referiremos al desasimiento o serenidad trabajado por Heidegger y propondremos a partir del concepto de la natalidad de Arendt la figura del apego. 


\section{Las tonalidades de un segundo inicio histórico}

El desasimiento o serenidad (Gelassenheitt) es un temple fundamental que opera en la dirección contraria a la figura dominante de nuestro tiempo, que es para Heidegger la época de la técnica en donde el cálculo y la manipulación son el modo de relación dominante con las cosas y entre nosotros. La mera formulación de una hipótesis, por ejemplo, dibuja claramente esa relación de dominio en donde un determinado pensamiento formula una verdad en la que irá sometiendo ámbitos de realidad al servicio de la comprobación de la hipótesis, la que es verdadera a partir de esta dominación. Cuando Heidegger nos habla de la técnica está entendiéndola como figura epocal, casi como un arquetipo que determina el modo en que nos aparecen las cosas y los otros, la técnica hace que cada cosa se nos presente primeramente como disponible para su consumo, como un recurso natural o humano, en este sentido, hasta el bosque más virgen se nos presenta técnicamente.

El desasimiento, por contraparte, es aquel tono, aquella actitud en la que nuestra relación con las cosas pretende justamente no ser dominante, no manipular al otro, para fundar desde allí otro modo de verdad; por eso se trata de desasimiento (más que de serenidad) porque consiste en "soltar" las relaciones de dominio, en no pretender que lo otro o el otro sea así como yo quiero que sea, y en ese sentido no me aparezca como algo meramente disponible. Por esta razón el desasimiento se aleja de la esperanza, al menos de esa esperanza con objeto determinado, con un telos, pues no fundamenta las relaciones con los otros a partir del cumplimiento o incumplimiento de determinadas expectativas, el desasimiento tiene que ver con el abandono de nuestras fijaciones respecto al futuro, aunque estas sean la esperanza en "un mundo mejor", eso ya sería una pretensión de manipulación. Pretender forzarla es un contrasentido, justamente el desasimiento apunta al no pretender forzar nada, ni siquiera el mismo desasimiento, porque no depende de nosotros que aparezca sino de un momento anterior que es el que se abra su posibilidad en el momento justo, es "una relación ... que no sostenemos sino que dejamos que 'eso' nos ocurra" (Gadamer, 2003: 190). El desasimiento es un modo de no-querer, no en el sentido de que en el desasimiento no se quiera "algo", o ninguna cosa, sino más bien en el sentido de "soltarse" del querer mismo, es allí donde se descansa, donde radica la calma. Habíamos dicho que el desasimiento no es una esperanza en el sentido de tener expectativas fijas para un futuro, aquí agregamos que sí contiene a la esperanza 
pero entendida como espera, como una actitud abierta a lo abierto pues "en la espera dejamos abierto aquello que esperamos, la espera no tiene propiamente objeto" (Heidegger, 1977: 44), el desasimiento aguarda confiadamente, pero no poniéndole condiciones al futuro.

La pregunta determinante para nuestro asunto es por qué el desasimiento sería capaz de reiniciar nuestras relaciones fundamentales desplazando las relaciones técnicas e inaugurando otro modo de construir nuestro mundo, por qué es una experiencia capaz de fundar historia. La respuesta, me parece, se encuentra en el carácter ontológico y negativo del desasimiento. Efectivamente el desasimiento desplaza la manipulación técnica, es una experiencia que niega la posibilidad de la manipulación, no a través de una necesidad lógica, sino que hace de la manipulación algo "no necesario", se deshace de esa necesidad. Pero eso es la sola negación de nuestro modo actual de estar en el mundo; falta, y es la propuesta que realizaremos aquí, el momento positivo, la fuerza, potencia y punto de apoyo capaz de gestar otro modo de configurar nuestro mundo.

Recordemos que en Heidegger los temples cumplen esa función de vinculatividad de sentido, y Heidegger se habría concentrado en el momento del cierre de posibilidades, presente ya en Ser y Tiempo (Heidegger, 1986, 235ss) en lo que conocemos como el ser para la muerte y la angustia, y presente también en el apremio, que es como hemos visto, una especie de angostura espacio-temporal donde el horizonte se cierra. El desasimiento por otra parte guarda esa negatividad en el "dejar ir", el soltar y el abandono. Pensamos que estos momentos al menos habrían de ser matizados o complementados, si es que mantenemos la pregunta por el poder iniciar un momento histórico, en lo que es la gestación de posibilidades, que propiamente puedan abrir e inaugurar un mundo.

Hannah Arendt piensa, en abierta crítica a las tesis heideggerianas, en la apertura de posibilidades y de la dimensión de la acción humana desde la natalidad, justamente como ese momento en que se puede reiniciar una historia. Klaus Held a propósito del asombro y su potencia para iniciar una historia le reclama a Heidegger no haber atendido a la natalidad: "Heidegger tuvo a la vista el carácter irruptivo del asombro sólo en esta, su forma decadente. Y de allí viene el hecho de que apenas haya visto el rasgo fundamental del asombro propio: el atemperamiento a la posibilidad de emprender un inicio. (...) Heidegger no pudo, en su interpretación del temple fundamental del inicio griego, ponerse a la altura de esta conservación del asombro propio, porque en su fenomenología del Dasein la natalidad no es tomada en cuenta en debida forma" (Held, 1988, 248ss). 
Arendt vincula estrechamente la natalidad a la acción ya que es esa la facultad de comenzar otra vez. "El milagro que salva al mundo, a la esfera de los asuntos humanos, de su ruina normal y 'natural' es en último término la natalidad, en el que se enraíza ontológicamente la facultad de la acción. Dicho en otras palabras, el nacimiento de nuevos hombres y un nuevo comienzo es la acción que son capaces de emprender los humanos por el hecho de haber nacido" (Arendt, 2012: 266). Destacamos, como se ve en esta cita, que la natalidad no sólo abre posibilidades, que es el ámbito donde Heidegger sitúa un inicio histórico, sino que determina la acción.

Pensando la figura de la natalidad como el poder iniciar, siguiendo el razonamiento heideggeriano de los temples fundamentales, podemos prestar atención específicamente a un momento crucial que comienza en el nacer y que la sicología llama apego. Sería posible realizar también la línea interpretativa de la natalidad vinculada al amor mundis trabajada por Arendt en sus textos sobre San Agustín (Arendt, 2001, 140ss) aunque aquí propondremos una tonalidad cercana pero con ciertos matices diferentes: el apego. El apego es entendido como la primera necesidad sicológica de quien viene al mundo que determina todos los otros vínculos y consiste en una especie de reconocimiento afectivo básico de que estamos con otro y nos confiamos a él, no al modo de un juicio analítico de que el otro es confiable sino que para el que ha nacido la dimensión del otro se abre a través de esa confianza y apoyo. Sin un apego inicial, o una reparación ulterior de su falta, difícilmente puede haber reconocimiento, el apego es su condición de posibilidad, abre la dimensión de la dignidad tanto propia como del "otro".

Otro carácter fundamental del apego es que se da exclusivamente a través del tacto. Podríamos decir incluso que el apego es un modo de tocar que se relaciona con la acogida, la contención y la calma. Este punto es determinante porque nos muestra además el estrechísimo vínculo entre nuestros cuerpos y los tonos afectivos. No se puede extirpar el cuerpo de una tonalidad sin alterarla radicalmente -que es lo que hace Heideggery en el apego es especialmente nítido: no hay apego sin tacto, por ello la dimensión de los otros no se puede abrir, al menos en un primer momento, a distancia. Y es que el tocar marca para el que ha nacido el primer límite con un afuera y en ese límite una proximidad, se toca la diferencia "con" otro. El tocar, nos dirá Jean-Luc Nancy, "acaricia, es esencialmente caricia, es decir que es deseo y placer de aproximar lo más posible una piel y de emplear esta proximidad para poner en juego las pieles una contra la otra" (Nancy, 2013: 19ss). El apego contiene esa caricia y también un mutuo acomodo, tono básico para poder apoyarnos en la realidad y con los otros. 
Si miramos desde el apego, como a contraluz lo que hemos dicho del desasimiento, podemos distinguir ciertos elementos cercanos entre el desasimiento y el poder iniciar de la natalidad; la contención, el cobijar y el dejarse confiar a la región del encuentro pueden ser pensados desde allí, pero difícilmente podemos pensar el desasimiento como un apego; el desasimiento es un soltarse al vacío, una apertura a lo abierto, al Entre mismo, no al con. Algo así como una natalidad desapegada y dejante, por no nombrar ese abandono que Heidegger mismo refiere al ser innumerables veces. Heidegger trabaja de diferentes formas una figura de contención (die Verhaltenheit) como momento del desasimiento, ella podría incluso ser pensada como una especie de "apego sin tacto, o apego frío", lo que es, como intentamos mostrar, un contrasentido.

Sin un apego básico parece imposible iniciar siquiera cualquier vía de reconocimiento, y en este sentido pensamos que podría cumplir un rol político importante a la hora de pensar el poder iniciar de la historia desde la natalidad.

\section{Conclusiones}

Hemos querido realizar un análisis de la cuestión de la banalidad del mal desde la perspectiva de la afectividad elaborada por Heidegger cruzando con ello de forma transversal varias cuestiones:

En términos generales hemos buscado realizar algunos trazos respecto a la función política de la afectividad y de un modo más preciso a la pregunta por las tonalidades posibles del mal banal y del llamado segundo inicio. Desde allí afirmamos que no se puede extraer la dimensión afectiva de la conformación histórica de las comunidades, ya que la afectividad es política en la medida que conforma el horizonte de posibilidades históricas. No se trata sólo de la afectividad como fenómeno subjetivo sino precisamente de aquel ámbito que está entre unos y otros y que en cierta medida posibilita su relación. Tampoco se trata de un irracionalismo ya que la afectividad es la forma del pensamiento, no hay pensamiento que no posea un tono.

A partir de esta tesis hemos propuesto que la banalidad del mal puede ser complementada con la dimensión afectiva, concretamente con el desprecio ya que la ira y el odio suponen un cierto reconocimiento del otro en cambio en el desprecio éste no aparece en cuanto tal. Del mismo modo seńalamos que el segundo inicio en Heidegger no está ligado a una solución forzada o violenta, 
como se insinúa a partir de las publicaciones de los cuadernos negros, ya que está pensado desde la tonalidad del desasimiento, que no es violento pero sí desapegado. Por esta razón proponemos finalmente repensar el segundo inicio junto a la tesis arendtiana de la natalidad y con la tonalidad del apego, la que además es corporal. Desde el apego sería posible quizá reelaborar la pregunta por volver a comenzar, otra vez, nuestra historia.

\section{Referencias bibliográficas}

Arendt Hannah (1999), Eichman y el Holocausto, Editorial Taurus, Madrid.

(2001), El concepto de amor en San Agustin, traducción y presentación de Agustín Serrano de Haro, Madrid, Editorial Encuentro.

(2002), La vida del espiritu, Editorial Paidós, Barcelona.

(2012), La condición bumana, Editorial Paidós, Buenos

Aires.

(1999), Los orígenes del totalitarismo, Editorial Taurus,

Madrid.

(2013), ¿Quées la politica?, Editorial Paidós, Buenos Aires.

Aristóteles (1999), Retórica, CEPC, Madrid.

Betancourt-Fornet Raul (2011), La filosofía intercultural y la dinámica del reconocimiento, Editorial Universidad Católica de Temuco, Temuco.

Bulo Valentina (2013), “Tabula rasa de los cuerpos”, en Revista La Cañada 4/6, 206-214, Santiago.

(2012), El temblor del ser: cuerpo y afectividad en la fllosofia de Martin Heidegger, Editorial Biblos, Buenos Aires.

Fédier François (2014) "Heidegger et le monde juif”, conferencia pronunciada el 24 de enero del 2014 en el Coloquio "Heidegger et les juifs"

Gadamer Hans-Georg (2003), Los caminos de Heidegger, Herder, Barcelona.

García de la Huerta (2015), Reseña Trawny Peter (2014), Heidegger et 
l'antisemitisme. Sur les “Cahiers noirs", en Revista de Filosofía n 71, pp 221-223.

Häsler Alfred (2002), El odio en el mundo actual, Editorial Alianza, Madrid. Heidegger Martin, (1977), Gelassenheit, Tubinga, Neske.

(1986), Seind und Zeit, Tubinga, Max Niemeyer.

(2014), Überlegungen XII-XV (Schwarze Hefte 19391941), Gesamtausgabe, Band 96, Frankfurt am Main, Vittorio Klostermann.

Held, Klaus, "Grundbestimmung und Zeitkritik bei Heidegger", Phaenomenologica, Heidegger et l'idée de la Phénoménologie, vol 108, p.248264, (1988), Kluwer Academic Publishers, Dordrecht-Boston-Londres.

Irigoyen Marie-France (1999), Acoso moral, el maltrato psicológico en la vida cotidiana, Editorial Paidós, Barcelona.

Nancy Jean-Luc (2013) Archivida, Ed. Quadrata, Traducción Marie Bardet y Valentina Bulo, Buenos Aires.

(2015), La banalité de Heidegger, Paris, Galilée.

Rancière Jacques, "El fin de la política o la utopía realista", En los bordes de lo político (2007), Ed. La Cebra, Buenos Aires.

Sánchez Cecilia, "Arendt e Irigaray: el lenguaje de la pluralidad y la intersubjetividad en las esferas de lo público y lo privado", Inédito.

Trawny Peter (2014), Heidegger et l'antisemitisme. Sur les "Cahiers noirs", Paris, Seuil. 